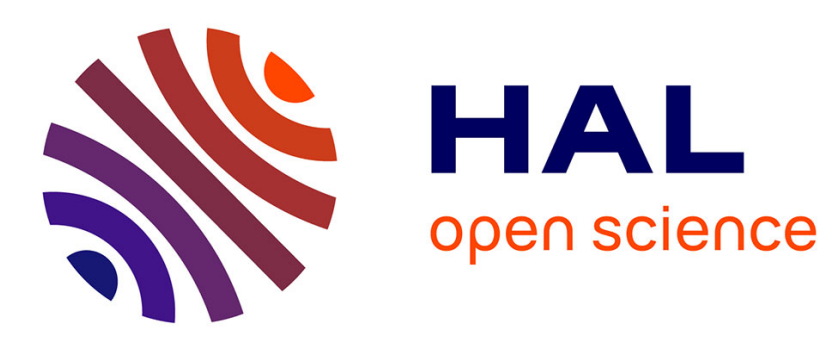

\title{
On shakedown of elastic-plastic bodies with temperature-dependent properties
}

\author{
Michaël Peigney
}

\section{To cite this version:}

Michaël Peigney. On shakedown of elastic-plastic bodies with temperature-dependent properties. VII European Congress on Computational Methods in Applied Sciences and Engineering (ECCOMAS 2016), Jun 2016, Crete Island, Greece. hal-01332892

\section{HAL Id: hal-01332892 \\ https://hal-enpc.archives-ouvertes.fr/hal-01332892}

Submitted on 16 Jun 2016

HAL is a multi-disciplinary open access archive for the deposit and dissemination of scientific research documents, whether they are published or not. The documents may come from teaching and research institutions in France or abroad, or from public or private research centers.
L'archive ouverte pluridisciplinaire HAL, est destinée au dépôt et à la diffusion de documents scientifiques de niveau recherche, publiés ou non, émanant des établissements d'enseignement et de recherche français ou étrangers, des laboratoires publics ou privés. 


\title{
ON SHAKEDOWN OF ELASTIC-PLASTIC BODIES WITH TEMPERATURE DEPENDENT PROPERTIES
}

\author{
Michaël Peigney \\ Université Paris-Est, Laboratoire Navier (UMR 8205), \\ CNRS, Ecole des Ponts ParisTech, IFSTTAR, F-77455 Marne la Vallée, France \\ e-mail: michael.peigney@enpc.fr
}

Keywords: Plasticity, Shakedown, Temperature-dependent properties, Melan's theorem

\begin{abstract}
For elastic-perfectly plastic structures under prescribed loading histories, the wellknown Melan's theorem gives a sufficient condition for shakedown to occur, i.e. for the evolution to become elastic in the large-time limit. The original Melan's theorem rests on the assumption that the material properties remain constant in time, independently on the applied loading. This communication addresses the long standing issue of extending Melan's theorem to temperaturedependent (or time-dependent) elastic moduli. The main motivation is to extend the range of applications of Melan's theorem to thermomechanical loading histories with large temperature fluctuations: In such case, the variation of the elastic properties with the temperature cannot be neglected. Some recent results obtained in perfect plasticity and viscoplasticity are presented and discussed.
\end{abstract}




\section{INTRODUCTION}

For elastic-perfectly plastic structures under prescribed loading histories, the well-known Melan's theorem [1, 2, 3] gives a sufficient condition for shakedown to occur, i.e. for the evolution to become elastic in the large-time limit. Intuitively, shakedown means that the plastic strain tends to a limit as time tends to infinity. The Melan's theorem has the distinctive property of being path-independent, i.e. independent on any residual stress that may exist initially in the structure. The shakedown theory has been the object of numerous developments, regarding both extensions of the original theorem to various nonlinear behaviors [4, 5, 6, 7, 8, 9] and numerical methods for assessing the shakedown limits in the case of parametrized loading histories [10, 11, 12, 13, 14, 15, 16].

This communication addresses the issue of extending Melan's theorem to temperature-dependent (or time-dependent) elastic moduli. The main motivation is to extend the range of applications of Melan's theorem to thermomechanical loading histories with large temperature fluctuations: In such case, the variation of the material properties with the temperature cannot be neglected. Whereas the case of temperature-dependent yield limits is well understood [17], the case of temperature-dependent elastic moduli remains a long standing issue and has been the object of several conjectures [18, 19, 20, 21]. The main difficulty is that the proof used in the original Melan's theorem - as well as in most of its knows extensions - crucially relies on some contraction properties that are lost when the elastic moduli are allowed to vary in time. A shakedown theorem has recently been proposed for elastic-perfectly plastic materials with time-periodic elastic properties [22]. The statement and proof of that theorem differ significantly from the case of constant material properties. A salient result is that time fluctuations of the elastic moduli need to remain small (in a certain sense) for shakedown to occur in a path-independent fashion.

In this communication, the theorem of [22] is presented and improved upon. The extension to elastic-viscoplastic materials is discussed.

\section{EVOLUTION EQUATION FOR THE STRESS FIELD}

Consider an elastic-plastic material occupying a domain $\Omega$ in the reference configuration. Under the small strains assumption, the strain $\varepsilon$, stress $\sigma$ and plastic strain $\varepsilon^{p}$ at position $x$ and time $t$ are related by the constitutive equations

$$
\begin{gathered}
\varepsilon(x, t)=L(x, t): \sigma(x, t)+\varepsilon^{\theta}(x, t)+\varepsilon^{p}(x, t), \\
\dot{\varepsilon}^{p}(x, t)=\phi^{\prime}(\sigma(x, t), x, t),
\end{gathered}
$$

where $\phi$ is the dissipation potential, assumed to be positive, convex, and null at the origin. In (1), $L$ is the (symmetric positive definite) elastic moduli tensor and $\varepsilon^{\theta}$ is the thermal strain tensor. Note the time dependence of $L, \varepsilon^{\theta}$ and $\phi$, which may result from imposed variations of the temperature. The elastic domain of the material is denoted by $\mathscr{C}(x, t)$, i.e. $\mathscr{C}(x, t)=\{\sigma$ : $\phi(\sigma, x, t)=0\}$.

Assuming quasi-static evolutions, the stress field $\sigma$ satisfies the equilibrium equations

$$
\operatorname{div} \sigma+f=0 \text { in } \Omega, \sigma \cdot n=T \text { on } \partial \Omega_{T},
$$

where $f(x, t)$ are body forces and $T(x, t)$ are tractions prescribed on a part $\partial \Omega_{T}$ of the boundary 
$\partial \Omega$. The strain field $\varepsilon$ and displacement field $u$ satisfy

$$
\varepsilon=\frac{1}{2}\left(\nabla u+\nabla^{T} u\right) \text { in } \Omega, u=v \text { on } \partial \Omega_{u}=\partial \Omega-\partial \Omega_{T}
$$

where $v(x, t)$ is a given function.

As usual in shakedown analysis, it is convenient to introduce the fictitious elastic response $\left(u^{E}, \sigma^{E}\right)$, i.e. the response of the system if it were purely elastic, defined by

$$
\begin{array}{r}
\varepsilon^{E}=L: \sigma^{E}+\varepsilon^{\theta}, \\
\operatorname{div} \sigma^{E}+f=0 \text { in } \Omega, \sigma^{E} \cdot n=T \text { on } \partial \Omega_{T}, \\
\varepsilon^{E}=\frac{1}{2}\left(\nabla u^{E}+\nabla^{T} u^{E}\right) \text { in } \Omega, u^{E}=v \text { on } \partial \Omega_{u} .
\end{array}
$$

The boundary value problem defined by Eqs. (1,4) governs the evolution of the fields $(u, \varepsilon, \sigma)$. An evolution equation involving only the stress field can be formulated from Eqs. (1,4), as is now detailed. Let $\mathcal{E}$ denote the space of stress fields, chosen as a subspace of symmetric second-order tensor fields with square-integrable components. The space $\mathcal{E}$ is equipped with the scalar product $\left\langle\sigma, \sigma^{\prime}\right\rangle=\int_{\Omega} \sigma(x): \sigma^{\prime}(x) d \omega$. The associated norm is denoted by \|\| , i.e. $\|\sigma\|=\sqrt{\langle\sigma, \sigma\rangle}$. For simplicity we assume in the following that $\mathcal{E}$ is of finite dimension.

Any stress field $\sigma$ satisfying (3) can be written as $\sigma=\sigma^{E}+\rho$ where $\rho$ belongs to the vectorial space $\mathcal{H} \subset \mathcal{E}$ of self-equilibrated stress fields, defined by

$$
\mathcal{H}=\left\{\rho \in \mathcal{E}: \operatorname{div} \rho=0 \text { in } \Omega, \rho \cdot n=0 \text { on } \partial \Omega_{T}\right\} .
$$

For an arbitrary $\rho^{\prime} \in \mathcal{H}$, it follows from $(1)$ that

$$
\int_{\Omega}\left(\rho^{\prime}-\rho\right): \frac{d\left(\varepsilon-\varepsilon^{E}\right)}{d t} d \omega=\int_{\Omega}\left(\rho^{\prime}-\rho\right): \frac{d}{d t}(L: \rho) d \omega+\int_{\Omega}\left(\rho^{\prime}-\rho\right): \phi^{\prime}(\sigma) d \omega .
$$

The principle of virtual power shows that the left-hand side of $(7)$ is equal to 0 , hence

$$
-\int_{\Omega}\left(\rho^{\prime}-\rho\right): \frac{d}{d t}(L(x, t): \rho) d \omega=\int_{\Omega}\left(\rho^{\prime}-\rho\right): \phi^{\prime}(\sigma, x, t) d \omega .
$$

Let $\Phi(\rho, t): \mathcal{H} \mapsto \mathbb{R}$ be the convex, positive function defined by

$$
\Phi(\rho, t)=\int_{\Omega} \phi\left(\sigma^{E}(x, t)+\rho(x), x, t\right) d \omega .
$$

Note that $\Phi(\cdot, t)$ vanishes on the set $\mathcal{K}(t)=\left\{\rho \in \mathcal{H}: \rho(x)+\sigma^{E}(x, t) \in \mathscr{C}(x, t) \forall x \in \Omega\right\}$. For later reference, note that $\mathcal{K}(t)=\mathcal{K}_{0}(t) \cap \mathcal{H}$ where

$$
\mathcal{K}_{0}(t)=\left\{\rho \in \mathcal{E}: \rho(x)+\sigma^{E}(x, t) \in \mathscr{C}(x, t) \forall x \in \Omega\right\} .
$$

We have (at least formally)

$$
\int_{\Omega}\left(\rho^{\prime}-\rho\right): \phi^{\prime}(\sigma) d \omega=\left\langle\rho^{\prime}-\rho, \Phi^{\prime}(\rho, t)\right\rangle .
$$

Further introducing the linear symmetric operator $\mathcal{L}(t): \mathcal{H} \mapsto \mathcal{H}$ defined by

$$
\left\langle\rho, \mathcal{L}(t) \rho^{\prime}\right\rangle=\int_{\Omega} \rho(x): L(x, t): \rho^{\prime}(x, t) d \omega \forall \rho, \rho^{\prime} \in \mathcal{H},
$$


Eq. (8) can be rewritten as

$$
-\frac{d}{d t}(\mathcal{L}(t) \rho)=\Phi^{\prime}(\rho, t)
$$

Since the elastic moduli tensor $L(x, t)$ is symmetric positive definite, it can easily be verified that $\mathcal{L}(t)$ is self-adjoint and positive definite. Its inverse is denoted by $\mathcal{M}(t)$. Starting from a given initial state $\rho(t=0)$, the evolution of the stress field in $\mathcal{H}$ is governed by the ordinary differential equation (9). The uniqueness of the stress rate $\dot{\rho}$ has been proved in [23].

In the case of time-independent elastic moduli, a change of scalar product allows Eq. (9) to be put in the form

$$
-\frac{d \rho}{d t}=\Phi^{\prime}(\rho, t)
$$

Properties of Eq. (10) are well understood. In particular, the distance between two solutions (as measured by the energy norm) is decreasing with time: For two solutions $\rho(t)$ and $\rho^{\prime}(t)$ of (10), we have indeed

$$
\frac{1}{2} \frac{d}{d t}\left\|\rho(t)-\rho^{\prime}(t)\right\|^{2}=\left\langle\dot{\rho}-\dot{\rho}^{\prime}, \rho-\rho^{\prime}\right\rangle=-\left\langle\Phi^{\prime}(\rho, t)-\Phi^{\prime}\left(\rho^{\prime}, t\right), \rho-\rho^{\prime}\right\rangle \leq 0
$$

where the convexity of $\Phi(\cdot, t)$ has been used. The contraction property (11) plays a crucial role when studying the asymptotic behaviour of solutions as $t \rightarrow+\infty$. If $\mathcal{L}(t)$ is a radial function of time, i.e. of the form

$$
\mathcal{L}(t)=f(t) \mathcal{L}^{0}
$$

then a change of variables allows Eq. (9) to be cast in the form (10) [22]. In the general case when $\mathcal{L}(t)$ is not radial, then the properties of Eq. (9) differ significantly from those of Eq. (10) - In particular, the contraction property is lost.

In the following it will be useful to measure 'how much' $\mathcal{L}(t)$ (or $\mathcal{M}(t)$ ) differs from a radial function. To that purpose, set

$$
\mu(t)=1 / \operatorname{tr} \mathcal{M}(t), \mathcal{M}_{0}(t)=\mu(t) \mathcal{M}(t)
$$

and define

$$
\gamma(a, b)=\int_{a}^{b}\left\|\dot{\mathcal{M}}_{0}(t)\right\| d t
$$

where $\|\cdot\| \cdot \|$ is the norm operator in $\mathcal{H}$, i.e. \|\| $\mathcal{M}(t)\left\|=\sup _{\rho^{\prime} \in \mathcal{H},\left\|\rho^{\prime}\right\|=1}\right\| \mathcal{M}(t) \rho^{\prime} \|$. Observe that $\gamma(a, v)$ vanishes if $\mathcal{L}(t)$ is of the form $(12)$ on the time interval $[a, b]$, and is non negative otherwise.

Remark: For simplicity, we have assumed so far that the dissipation potential $\phi$ is differentiable, which is the case for viscoplasticity. For perfect plasticity, the dissipation potential is only subdifferentiable: Eqs. (9) and (10) have to be replaced with

$$
-\frac{d}{d t}(\mathcal{L}(t) \rho)=\partial I_{\mathcal{K}(t)}(\rho, t)
$$

and

$$
-\frac{d \rho}{d t}=\partial I_{\mathcal{K}(t)}(\rho, t)
$$

respectively. In Eqs $\sqrt{14} 15,, I_{\mathcal{K}(t)}$ is the indicator function of the set $\mathcal{K}(t)$ and $\partial$ denotes the subdifferential operator. Eq. [15] corresponds to the so-called 'sweeping process' [24]. 


\section{SHAKEDOWN THEOREM}

In the following we study the asymptotic behavior of solutions to (9) as $t \rightarrow \infty$. We consider the case where $\mathcal{L}(t), \mathcal{K}(t)$ are periodic in time (with the same period $T$ ) and assume that $\mathcal{K}_{0}(t)$ and $\mathcal{L}(t)$ are bounded by some constants denoted respectively by $M$ and $C$, i.e.

$$
\left\|\rho^{\prime}\right\| \leq M,\|\mathcal{L}(t)\| \leq C
$$

for all $t$ and $\rho^{\prime} \in \mathcal{K}_{0}(t)$. As mentioned in the introduction, shakedown corresponds to the situation where the evolution becomes elastic in the large time limit. Elastic evolutions are characterized by the fact that $\phi(\sigma(x, t), x, t)=0$. Concerning the evolution equation 9 ) for the stress field, it follows that the distinctive properties of an elastic solution $\rho^{*}(t)$ are

$$
\frac{d}{d t}\left(\mathcal{L}(t) \rho^{*}(t)\right)=0, \rho^{*}(t) \in \mathcal{K}(t) \forall t \in[0, T] .
$$

Shakedown corresponds to the situation where the residual stress converges towards an elastic solution as $t \rightarrow \infty$. Our main objective is to examine conditions under which every solution of (9) converges towards an elastic solution in the large time limit. More precisely, the main result we discuss is the following

Shakedown theorem: If

(i) There exists an elastic solution $\rho^{*}$ to Eq. (9) and some $r>0$ such that

$$
\forall \rho^{\prime} \in \mathcal{H}, \forall t \in[0, T],\left\|\rho^{\prime}-\rho^{*}(t)\right\| \leq r \Longrightarrow \rho^{\prime} \in \mathcal{K}(t) ;
$$

(ii) the elastic moduli are such that

$$
\frac{\gamma(0, T)}{\inf \mu}<\frac{r}{2 C M}
$$

then shakedown occurs, whatever the initial state is.

That theorem holds for elastic-perfectly plastic materials as well as for Duvaut-Lions elastoviscoplastic materials (see Sect. 3.3). Condition $(i)$ means that there exists an elastic solution that remains in the interior of the elasticity domain $\mathcal{K}(t)$ : It is a reformulation of the Melan's condition found in the classical Melan's theorem. Condition (ii) sets restriction on the time variations of the elastic moduli. Setting such a restriction is necessary to obtain global convergence results: One can indeed find counterexamples in which condition $(i)$ is fulfilled and some solutions of (9) do not become elastic in the large time limit [22].

\subsection{Variation of the elastic energy}

Let $\rho^{*}(t)$ be an elastic solution satisfying $(i)$ and let $\rho(t)$ denotes an arbitrary solution of 9 ). We consider the positive function $f$ defined as

$$
f(t)=\frac{1}{2}\left\langle\tau(t), \mathcal{L}_{0}(t) \tau(t)\right\rangle .
$$

where $\tau(t)=\mu(t)\left(\rho(t)-\rho^{*}(t)\right)$. The function $f$ is referred to as the elastic energy. The shakedown theorem stated above rests critically on the property

$$
f(a+T)-f(a) \leq(2 N \gamma(0, T)-r \inf \mu) \int_{a}^{a+T}\|\dot{\eta}(t)\| d t
$$


where

$$
N=\sup _{a \leq t \leq a+T}\left\{\|\mathcal{L}(t) \rho(t)\|,\left\|\mathcal{L}(t) \rho^{*}(t)\right\|\right\} .
$$

Eq. (19) bounds the variation of the elastic energy over a cycle. That bound plays the role of the contraction property that holds only in the case of time-independent elastic moduli. Observe in particular from 19 ) that $f(a+T)-f(a) \leq 0$ if $\gamma(0, T)$ is sufficiently small.

The rest of this Section is devoted to a proof of Eq. (19). The inequality (19) is obtained by performing minor modifications to a similar result obtained in [22] for perfect plasticity, hence we only sketch the main steps of the proof and refer to [22] for details. Recalling that $d\left(\mathcal{L}(t) \rho^{*}(t)\right) / d t=0$ and setting $H(t)=-\frac{1}{2}\left\langle\tau(t), \dot{\mathcal{L}}_{0}(t) \tau(t)\right\rangle$, some straightforward manipulation shows that

$$
\dot{f}(t)=\langle\tau(t), \dot{\eta}(t)\rangle+H(t)
$$

where $\eta(t)=\mathcal{L}(t) \tau(t)$. Integrating on the time interval $[a, a+T]$ gives

$$
f(a+T)-f(a)=\int_{a}^{a+T}\langle\tau(t), \dot{\eta}(t)\rangle d t+\int_{a}^{a+T} H(t) d t .
$$

In the following, the two integrals in the right-hand side of Eq. (20) are bounded separately. Let us first consider the integral $\int_{a}^{a+T}\langle\tau(t), \dot{\eta}(t)\rangle d t$. Noting from 99 that $\dot{\eta}(t)=-\Phi^{\prime}(\rho(t), t)$ and recalling that $\Phi$ is convex, positive and vanishes in $\mathcal{K}(t)$, we have

$$
0 \leq \Phi(\rho(t), t) \leq\left\langle\dot{\eta}(t), \rho^{\prime}(t)-\rho(t)\right\rangle \text { for all } \rho^{\prime} \in \mathcal{K}(t) .
$$

Condition $(i)$ implies that $\rho^{*}(t)-r \dot{\eta} /\|\dot{\eta}\|$ is in $\mathcal{K}(t)$. Hence 21) gives $0 \leq\left\langle\dot{\eta}(t), \rho^{*}(t)-\right.$ $\left.r \frac{\dot{\eta}}{\|\dot{\eta}\|}-\rho(t)\right\rangle$ i.e. $\left\langle\dot{\eta}(t), \rho(t)-\rho^{*}(t)\right\rangle \leq-r\|\dot{\eta}(t)\|$. Therefore we obtain

$$
\int_{a}^{a+T}\langle\tau(t), \dot{\eta}(t)\rangle d t \leq-r(\inf \mu) \int_{a}^{a+T}\|\dot{\eta}\| d t
$$

Now consider the integral $\int_{a}^{a+T} H(t) d t$. We have $\tau(t)=\mathcal{L}_{0}^{-1}(t)(\eta(t)-\eta(a)+s)$ where $s=\mathcal{L}(a)\left(\rho(a)-\rho^{*}(a)\right)$ is independent on $t$. Some manipulations (see [22]) show that

$$
\int_{a}^{a+T} H(t) d t \leq \frac{1}{2} \int_{a}^{a+T}\|\eta(t)-\eta(a)\| \cdot\left\|\dot{\mathcal{M}}_{0}(t)\right\| \cdot\|\eta(t)-\eta(a)+2 s\| d t .
$$

Since $\eta(t)-\eta(a)+2 s=\mathcal{L}(t) \tau(t)+\mathcal{L}(a) \tau(a)-2 \mathcal{L}(a) \rho^{*}(a)$, we have

$$
\|\eta(t)-\eta(a)+2 s\| \leq 4 N .
$$

We also have, for $t \in[a, a+T]$,

$$
\|\eta(t)-\eta(a)\|=\left\|\int_{a}^{t} \dot{\eta}\left(t^{\prime}\right) d t^{\prime}\right\| \leq \int_{a}^{t}\left\|\dot{\eta}\left(t^{\prime}\right)\right\| d t^{\prime} \leq \int_{a}^{a+T}\left\|\dot{\eta}\left(t^{\prime}\right)\right\| d t^{\prime} .
$$

Substituting 24,25) in 23) and using the definition 13 of $\gamma(a, b)$, we obtain

$$
\int_{a}^{a+T} H(t) d t \leq 2 N \gamma(a, a+T) \int_{a}^{a+T}\|\dot{\eta}(t)\| d t .
$$

Replacing (22,26) in (19) and observing by periodicity that $\gamma(a, a+T)=\gamma(0, T)$, Eq. (19) is obtained. 


\subsection{Case of elastic-perfectly plastic materials}

In perfect plasticity, the stress is constrained to remain in the elasticity domain $\mathscr{C}(x, t)$, which by (16) implies that $\|\rho(t)\| \leq M$ and $\left\|\rho^{*}(t)\right\| \leq M$ for all $t$. By (16) we thus have $\|\mathcal{L}(t) \rho(t)\| \leq$ $C M$ and $\left\|\mathcal{L}(t) \rho^{*}(t)\right\| \leq C M$. In (19), the constant $N$ thus satisfies $N \leq C M$. Applying Eq. (19) on the time interval $[i T,(i+1) T]$ gives

$$
f((i+1) T)-f(i T) \leq-m \int_{i T}^{(i+1) T}\|\dot{\eta}(t)\| d t
$$

where $m=r(\inf \mu)-2 C M \gamma(0, T)$ is non-negative by (ii). Summing over $i=0, \cdots, I$ gives

$$
\int_{0 T}^{I T}\|\dot{\eta}(t)\| d t \leq \frac{1}{m} f(0)
$$

Taking the limit $I \rightarrow \infty$ shows that the integral $\int_{0}^{S}\|\dot{\eta}(t)\| d t$ converges as $S \longrightarrow+\infty$. It follows that $\eta(t)$ also converges towards a limit $\eta_{\infty}$ as $t \longrightarrow+\infty$. Setting $\rho_{\infty}(t)=\mathcal{M}(t) \eta_{\infty}$, it can be checked that $\rho(t)-\rho_{\infty}(t) \longrightarrow 0$ as $t \rightarrow \infty$, and that $\rho_{\infty}(t)$ is an elastic solution.

Remark: For perfect plasticity, the theorem stated above slightly improves on the result obtained in [22]. In [22], a condition analog to (ii) was formulated, with the factor 2 in (ii) replaced by a factor 3 .

\subsection{Case of elastic-viscoplastic materials}

Compared to perfect plasticity, an additional difficulty is that the stress is not restricted to remain in the elasticity domain: There is no direct bound on $\rho(t)$, hence no direct on the constant $N$ in (19) . That difficulty, however, can be overcome in the case of a Duvaut-Lions viscoplastic behavior, i.e. for a dissipation potential of the form

$$
\phi(\sigma, x, t)=\frac{\alpha}{2}\left|\sigma-P_{\mathscr{C}(x, t)} \sigma\right|^{2}
$$

where $\alpha>0$ is a viscosity parameter (assumed to be independent on $(x, t)$ for simplicity) and $P_{\mathscr{C}(x, t)}$ denotes the projection on the elasticity domain $\mathscr{C}(x, t)$. Full details will be published elsewhere [25], but the main point is that $\eta(t)=\mathcal{L}(t) \rho(t)$ is bounded by a constant $N^{\prime}$ for large time. Moreover, that constant $N^{\prime}$ can be chosen as arbitrary close to $C M$. Using (19) with a value of $N^{\prime}$ such that $C M<N^{\prime}<(r \inf \mu) / 2 \gamma(0, T)$, one can show that $\eta(t)$ converges as $t \rightarrow \infty$ and consequently that $\rho(t)$ converges towards an elastic solution (the reasoning is similar to that presented in Sect. 3.2.

\section{EXAMPLE}

As an illustrative example, consider the 3-bar truss represented in Fig. 1. We denote by $\sigma_{i}$, $\varepsilon_{i}, \varepsilon_{i}^{p}$ the uniaxial stress, total strain and plastic strain in bar $i$. The constitutive relation in each bar reads as

$$
\varepsilon_{i}=\frac{\sigma_{i}}{E_{i}(t)}+\varepsilon_{i}^{p}+\varepsilon_{i}^{\theta}, \dot{\varepsilon}_{i}^{p}=\phi^{\prime}\left(\sigma_{i}\right)
$$

The truss is stress-free, i.e. $\sigma_{1}+\sigma_{2}+\sigma_{3}=0$. The loading consists of a thermal strain $\varepsilon_{2}^{\theta}(t)=$ $a \sin (2 \pi t / T)$ in bar 2. We assume that $E_{1}=E_{3}$ remains independent on time and that $E_{2}(t)$ vary as $E_{2}(t)=E /\left(1+\delta \sin ^{2}(\pi t / T)\right)$. The elasticity domain is the same for all bars and taken as $\left[-\sigma_{y}, \sigma_{y}\right]$ where the yield limit $\sigma_{y}$ is independent on temperature. 


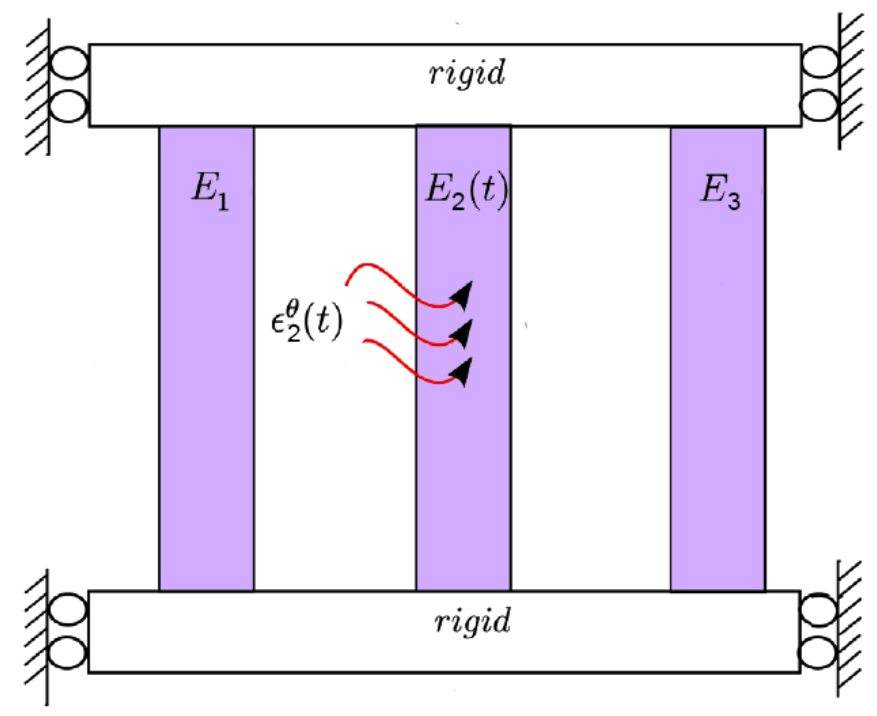

Figure 1: Three-bar truss with large thermal loading.

For the problem at hand, the vectorial space $\varepsilon$ of stress fields identifies with $\mathbb{R}^{3}$ and is equipped with the scalar product $\left\langle\sigma, \sigma^{\prime}\right\rangle=\sum_{i=1}^{3} \sigma_{i} \sigma_{i}^{\prime}$. Writing the stress state of the system as $\sigma=\left(\sigma_{1}, \sigma_{2}, \sigma_{3}\right)$, the fictitious elastic response of the system is

$$
\sigma^{E}(t)=E \frac{\varepsilon_{2}^{\theta}(t)}{3+2 \delta \sin ^{2}(\pi t / T)}(1,-2,1) .
$$

It follows that $\left\|\sigma^{E}(t)\right\| \leq \sqrt{6} \sigma^{\theta} / 3$ where $\sigma^{\theta}=E \sup _{t}\left|\varepsilon_{2}^{\theta}(t)\right|$. Any $\sigma$ in $\mathcal{K}^{0}$ verifies $\left\|\sigma+\sigma^{E}\right\| \leq$ $\sqrt{3} \sigma_{y}$. Hence the constant $M$ in $(16)$ can be taken as

$$
M=\sqrt{3} \sigma_{y}+\sqrt{6} \sigma^{\theta} / 3
$$

In the present case, the space $\mathcal{H}$ of self-equilibrated stress fields is of dimension 2. Calculating the operator $\mathcal{L}(t)$ shows that

$$
C=\frac{3+2 \delta}{3 E}, \gamma(0, T)=\frac{\delta}{3+\delta}, \inf \mu=\frac{1}{2 E} .
$$

Note that $(0,0,0)$ is an elastic solution. As long as $\sigma^{\theta} / \sigma_{y} \leq 3 / 2$, it can be verified that $(0,0,0)$ satisfies condition $(i)$ with a value or $r$ given by

$$
r=\left(\frac{\sigma_{y}}{2}-\frac{\sigma^{\theta}}{3}\right) \sqrt{6}
$$

In Fig. 2 are represented the values $\left(\sigma^{\theta}, \delta\right)$ satisfying conditions $(i)$-(ii): Shakedown occurs for any $\left(\sigma^{\theta}, \delta\right)$ below the solid line shown in Fig. 2. For $\delta=0$, we obtain that shakedown occurs provided that $\sigma^{\theta} \leq 3 / 2 \sigma_{y}$, in agreement with the classical Melan's theorem for timeindependent moduli. As can be observed on Fig. 2, the time fluctuations of the elastic moduli result in a decrease of the shakedown limit on $\sigma^{\theta}$. Those results apply both to elastic-perfectly plastic materials and to elasto-viscoplastic material of the Duvaut-Lions type. 


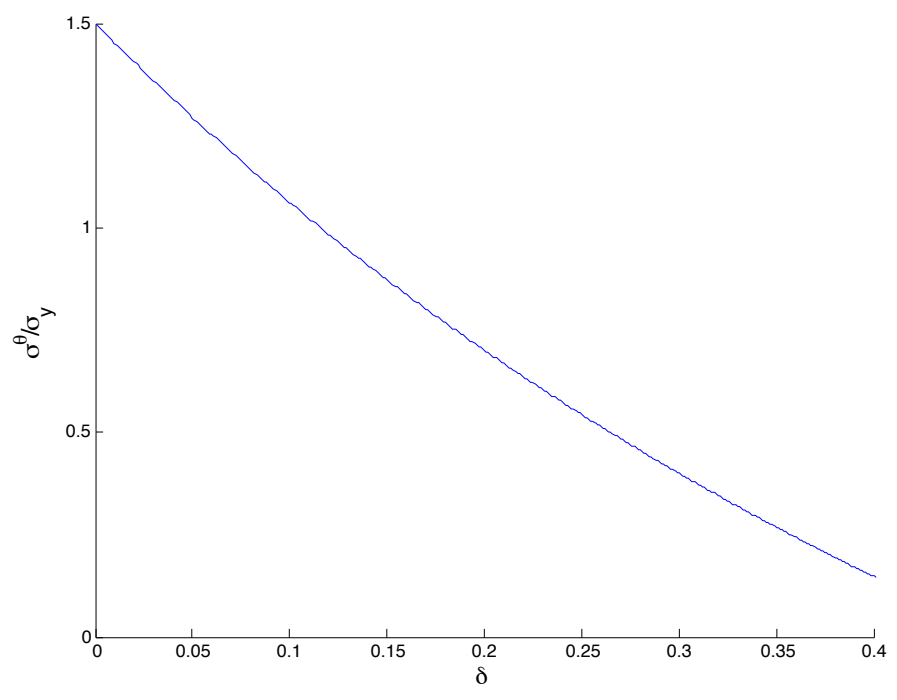

Figure 2: Shakedown limit for the three-bar truss.

In Fig. 3 are plotted the incremental evolution of the plastic strain and residual stress in bar 2, starting from an initial residual stress $\rho(0)=(0.46,-0.53,0.07) E$. The plots on the left correspond to a elastic-perfectly plastic behavior, while the plots on the right correspond to a elastic-viscoplastic behavior. We set $\delta=0.1$ and $\varepsilon^{\theta}(t)=0.1\left(\sigma_{y} / E\right) \sin (2 \pi t / T)$ : Such values are below the shakedown limit shown in Fig. 2. As expected from the shakedown theorem, we observe the convergence of the plastic strain towards a stabilized value. In the case of perfect plasticity, that convergence is reached after the first loading cycle. In contrast,the convergence is slow in the case of viscoplasticity. Observe that the residual stress does not converge towards a stabilized value: This is a direct consequence of the time dependence of the elastic moduli.
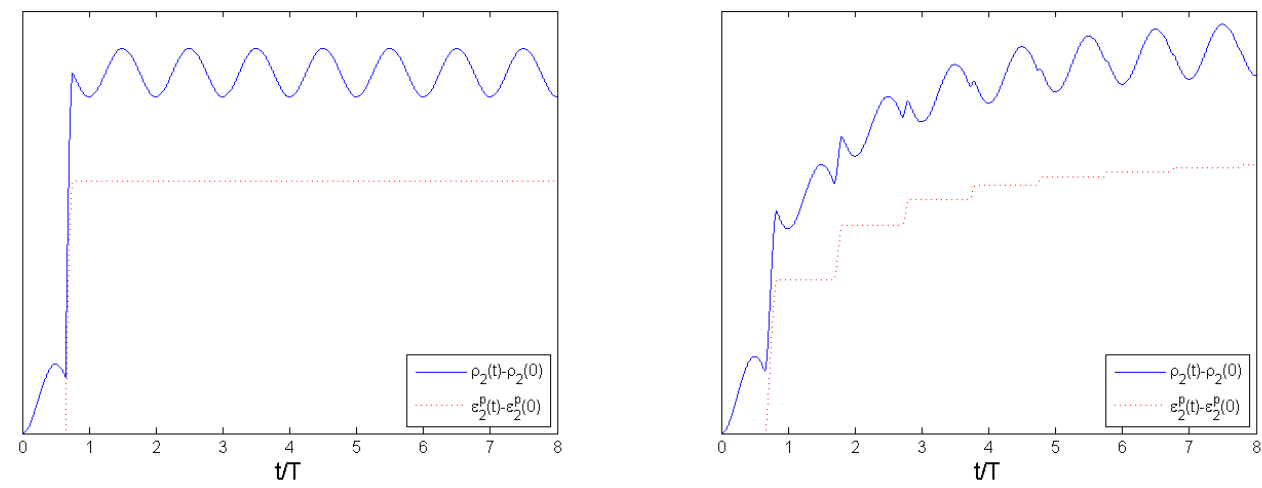

Figure 3: Residual stress and plastic strain in bar 2: (left) elastic-perfectly plastic material; (right) elasticviscoplastic material

\section{REFERENCES}

[1] E. Melan, Theorie statisch unbestimmter systeme aus ideal-plastischen baustoff, Sitz.Berl.Ak.Wiss. 145 (1936) 195-218. 
[2] P. S. Symonds, Shakedown in continuous media, J. Appl. Mech. 18 (1951) 85-89.

[3] W. T. Koiter, General problems for elastic solids, Progress in solid mechanics (1960).

[4] D. C. Pham, On shakedown theory for elastic-plastic materials and extensions, Journal of the Mechanics and Physics of Solids 56 (2008) 1905-1915.

[5] Q. S. Nguyen, On shakedown analysis in hardening plasticity, Journal of the Mechanics and Physics of Solids 51 (2003) 101-125.

[6] Y. J. Ahn, E. Bertocchi, J. Barber, Shakedown of coupled two-dimensional discrete frictional systems, Journal of the Mechanics and Physics of Solids 56 (12) (2008) 3433-3440.

[7] M. Peigney, Shakedown theorems and asymptotic behaviour of solids in non-smooth mechanics, Eur. J. Mech. A 29 (5) (2010) 784-793.

[8] M. Peigney, On shakedown of shape memory alloys structures, Annals of Solid and Structural Mechanics 6 (2014) 17-28.

[9] D. Weichert, A. Ponter, A historical view on shakedown theory, in: E. Stein (Ed.), The History of Theoretical, Material and Computational Mechanics-Mathematics Meets Mechanics and Engineering, Springer, 2014, pp. 169-193.

[10] J. Zarka, J. Frelat, G. Inglebert, A new approach to inelastic analysis of structures, Martinus Nijhoff Publishers, Dordrecht (1988).

[11] H. Maitournam, B. Pommier, J. Thomas, Détermination de la réponse asymptotique d'une structure anélastique sous chargement cyclique, C.R. Mecanique 330 (2002) 703-708.

[12] V. Carvelli, Z. Cen, Y. Liu, G. Maier, Shakedown analysis of defective pressure vessels by a kinematic approach, Archive of Applied Mechanics 69 (9-10) (1999) 751-764.

[13] M. Peigney, C. Stolz, Approche par contrôle optimal des structures élastoviscoplastiques sous chargement cyclique, C.R.Acad.Sci. Paris, II 329 (2001) 643-648.

[14] M. Peigney, C. Stolz, An optimal control approach to the analysis of inelastic structures under cyclic loading, Journal of the Mechanics and Physics of Solids 51 (2003) 575-605.

[15] J.-W. Simon, D. Weichert, Shakedown analysis of engineering structures with limited kinematical hardening, International Journal of Solids and Structures 49 (15) (2012) 21772186.

[16] K. V. Spiliopoulos, K. D. Panagiotou, A direct method to predict cyclic steady states of elastoplastic structures, Computer Methods in Applied Mechanics and Engineering 223 (2012) 186-198.

[17] G. Borino, Consistent shakedown theorems for materials with temperature dependent yield functions, International Journal of Solids and Structures 37 (22) (2000) 3121-3147.

[18] J. König, A shakedown theorem for temperature dependent elastic moduli, Bull. Ac. Pol. Sci., Ser. Sci. Techn. 17 (3) (1969) 161-165. 
[19] B. Halphen, S. di Domizio, Evolution des structures élastoplastiques dont les coefficients d'élasticité dépendent de la température, 17ème congrès français de mécanique, Troyes (2005).

[20] S. Hasbroucq, A. Oueslati, G. De Saxcé, Inelastic responses of a two-bar system with temperature-dependent elastic modulus under cyclic thermomechanical loadings, International Journal of Solids and Structures 47 (14) (2010) 1924-1932.

[21] S. Hasbroucq, A. Oueslati, G. De Saxcé, Analytical study of the asymptotic behavior of a thin plate with temperature-dependent elastic modulus under cyclic thermomechanical loadings, Int. J. Mech. Sci. 54 (1) (2012) 95-104.

[22] M. Peigney, Shakedown of elastic-perfectly plastic materials with temperature-dependent elastic moduli, Journal of the Mechanics and Physics of Solids 71 (2014) 112-131.

[23] B. Halphen, Elastic perfectly plastic structures with temperature dependent elastic coefficients, C.R. Mécanique 333 (8) (2005) 617-621.

[24] J. J. Moreau, Evolution problem associated with a moving convex set in a hilbert space, J. Diff. Eq. 26 (3) (1977) 347-374.

[25] M. Peigney, On Melan's theorem in temperature-dependent viscoplasticity, to appear (2016). 